\title{
Lettre de Jean Wahl à Martin Heidegger
}

Ian Alexander Moore et Barbara Wahl, eds.

Journal of French and Francophone Philosophy - Revue de la philosophie française et de langue française, Vol XXIX, No 1-2 (2021) pp 169-172

\author{
Vol XXIX, No 1-2 (2021) \\ ISSN 1936-6280 (print) \\ ISSN 2155-1162 (online) \\ DOI $10.5195 /$ jffp. 2021.993 \\ www.jffp.org
}

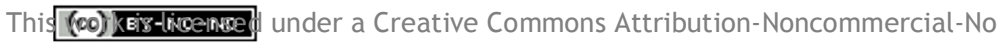
Derivative Works 3.0 United States License.

\section{ULIS D-Sunk}

This journal is operated by the University Library System of the University of Pittsburgh as part of its D-Scribe Digital Publishing Program, and is co-sponsored by the University of Pittsburgh Press 


\title{
Lettre de Jean Wahl à Martin Heidegger
}

\author{
Ian Alexander Moore \\ Loyola Marymount University
}

\author{
Barbara Wahl \\ Independent Scholar
}

\section{Préface}

Cette lettre, publiée ici pour la première fois en français, dans sa version originale, a été envoyée par Jean Wahl à Martin Heidegger le 12 décembre 1937. Elle répond à une lettre que Heidegger avait écrite à Wahl une semaine plus tôt au sujet des thèses de Wahl dans la célèbre conférence «Subjectivité et transcendance ${ }^{1} »$. Dans cette conférence, qui a été décrite comme "un tournant dans l'histoire intellectuelle du XXe siècle ${ }^{2}$ ", Wahl s'interrogeait, entre autres, sur la mesure dans laquelle la philosophie pouvait fournir une théorie générale des intuitions d'existences particulières telles que Kierkegaard ou Nietzsche. En d'autres termes, une philosophie de l'existence était-elle possible, ou ces existences elles-mêmes n'étaient-elles pas «à la fois plus 'existentielles' et plus vraiment philosophiques que les philosophies de l'existence $^{3}$ » ? Dans sa réponse, Heidegger déclarait que sa propre pensée ne pouvait être qualifiée de philosophie de l'existence, car elle ne s'occupait de l'être humain que dans la mesure où celui-ci pouvait éclairer l'être lui-même (das Sein). Wahl, cependant, n'était pas convaincu, et dans la lettre publiée ici, il défend à la fois sa décision d'appeler Heidegger un philosophe de l'existence et propose plusieurs objections à la manière dont Heidegger essaie de répondre à la question de l'être.

(This letter, published here in the original French for the first time, was sent by Jean Wahl to Martin Heidegger on December 12, 1937. It responds to a letter that Heidegger had written to Wahl a week earlier about the latter's theses in the celebrated lecture "Subjectivity and Transcendence." In this 
lecture, which has been called "a turning point in twentieth-century intellectual history," Wahl questioned, among other things, the extent to which philosophy could provide a general theory of the insights of particular existences such as Kierkegaard or Nietzsche. Was, in other words, a philosophy of existence possible, or were not these existences themselves "more 'existential' and more truly philosophical than the philosophies of existence?" In his reply, Heidegger claimed that his own thought could not be labeled a philosophy of existence, for it concerned itself with the human being only insofar as the latter could shed light on being itself (das Sein). Wahl, however, was not convinced, and in the letter published here he both defends his decision to call Heidegger a philosopher of existence and offers several objections to the way in which Heidegger tries to answer the question of being.)

\section{Avenue du Colonel Bonnet}

Paris $16^{\circ}$

12 décembre [1937]

Monsieur et cher collègue,

J'ai été très heureux de recevoir votre lettre et vous en remercie vivement ${ }^{4}$.

Je me reprochais d'avoir mal dit dans ces courtes lignes toute l'admiration que j'éprouve pour votre œuvre, d'avoir insisté surtout sur des critiques ou des réserves. J'ai eu l'occasion, à la fin de ma communication orale, de dire quelle importance j'attachais à « Sein und Zeit » ainsi qu'à vos conférences ${ }^{5}$.

Que votre philosophie soit ou ne soit pas Existenzphilosophie, la question m'importe à moi-même assez peu. - La philosophie de Platon est en un sens Existenzphilosophie. La mort d'un homme qui est Socrate, et son existence, est son centre.

Il m'est difficile de penser que ce que vous dites de la mort, de l'angoisse, ne puisse être rapproché de la philosophie de l'existence. En ce sens, je ne me sens pas coupable, - quand je vous ai rangé auprès des philosophes de l'Existence.

Mais je reconnais que ce serait un tort grave de la part des critiques, de ne pas voir, comme vous l'indiquez fortement dans votre lettre, que votre question est celle de l'être. 
Ce que je vois encore assez mal, c'est de quelle façon vous la résolvez. Je vois bien qu'il y a une relation entre l'être et le néant, relation - si je comprends bien - de transcendance de l'être par rapport au néant ; - en même temps peut-être d'immanence du néant à l'être ; puisque tout être est comme pénétré par le néant, - par le fait du temps et de l'être-pour-la-fin.

La question de l'être est donc transposée, - toujours si je comprends bien - dans celle du temps et du néant.

Pour le temps, j'admire beaucoup certaines des choses sur ses trois extases. Mais quant à la Wiederholung, je crois, comme je l'ai indiqué dans ma conférence $^{6}$, que c'est plutôt une volonté, - volonté métaphysique de puissance, - une idée de la volonté, (et finalement un succédané de l'éternité) qu'une réalité.

Quant au néant, je dois dire que grand admirateur de Boehme, j'ai été toujours très attiré par l'idée d'affirmer l'être du rien. - Mais d'autre part les objections de Platon et l'idée de la synonymie d'altérité et du néant (idées reprises et précisées par Bergson) me frappent beaucoup.

De sorte que je me dis que l'idée de néant est un mythe. J'aimerais y croire. Je ne parviens cependant pas à être persuadé par votre étude : «Qu'estce que la métaphysique? », quelque grande admiration que j'éprouve pour elle.

J'aimerais pouvoir un jour m'entretenir avec vous. Mais toutes sortes d'obstacles s'y opposent pour le moment.

Sachez du moins, Monsieur et cher collègue, que je vous suis profondément reconnaissant de m'avoir écrit, d'avoir précisé la direction de pensée qu'il faut suivre pour interpréter votre œuvre, sachez aussi quelle place éminente je vous attribue parmi les philosophes contemporains, et veuillez agréer je vous prie, l'expression de mes sentiments d'entier dévouement.

Jean Wahl

J'ai écrit il y a quelques années une étude intitulée «Heidegger et Kierkegaard » qui a paru dans les Recherches Philosophiques et que je pourrais vous envoyer si cela vous intéressait ${ }^{7}$. Mais pour le moment, je ne l'ai qu'en épreuves. D'ailleurs, elle ne me satisfait plus, et j'aimerais pouvoir un jour la compléter et la préciser. 
${ }^{1}$ [Jean Wahl, « Subjectivité et transcendance, » Bulletin de la Société Française de Philosophie 37, no 5 (Octobre-Décembre 1937) : 161-211. Republié, avec des omissions et une addition, Jean Wahl, Existence humaine et transcendance (Neuchâtel : Éditions de la Baconnière, 1944), 39-51, 113-59. Pour une discussion, voir Jean Wahl, Transcendence and the Concrete, éd. Alan D. Schrift and lan Alexander Moore (New York : Fordham University Press, 2017), 152-56, 213.]

2 Samuel Moyn, Origins of the Other : Emmanuel Levinas between Revelation and Ethics (Ithaca, NY : Cornell University Press, 2005), 182.

${ }^{3}$ Wahl, «Subjectivité et transcendance, » 163 ; Existence humaine et transcendance, 41.

${ }^{4}$ Dans « Subjectivité et transcendance, » 193 ; Existence humaine et transcendance, 134-35.

5 Wahl, «Subjectivité et transcendance, » 172 ; Existence humaine et transcendance, 51.

6 Wahl, «Subjectivité et transcendance, » 162 ; Existence humaine et transcendance, 41.

7 Wahl, « Heidegger et Kierkegaard : Recherche des éléments originaux de la philosophie de Heidegger, » Recherches Philosophiques 2 (1932-1933) : 349-70. Republié, avec des révisions mineures, dans Wahl, Études kierkegaardiennes (Paris : Fernand Aubier, 1938), 455-76. 